\title{
Community involvement in the prevention of mother-to-child transmission of HIV: Insights and recommendations
}

\author{
Naomi Rutenberg \\ Population Council \\ Mary Lyn Field-Nguer \\ Laura Nyblade
}

Follow this and additional works at: https://knowledgecommons.popcouncil.org/departments_sbsr-hiv

Part of the Community Health and Preventive Medicine Commons, Family, Life Course, and Society Commons, and the International Public Health Commons

How does access to this work benefit you? Let us know!

\section{Recommended Citation}

Rutenberg, Naomi, Mary Lyn Field-Nguer, and Laura Nyblade. 2001. "Community involvement in the prevention of mother-to-child transmission of HIV: Insights and recommendations," Community Involvement in Initiatives to Prevent Mother-to-Child Transmission of HIV: A Collaborative Project. New York and Washington, DC: Population Council and International Center for Research on Women. 


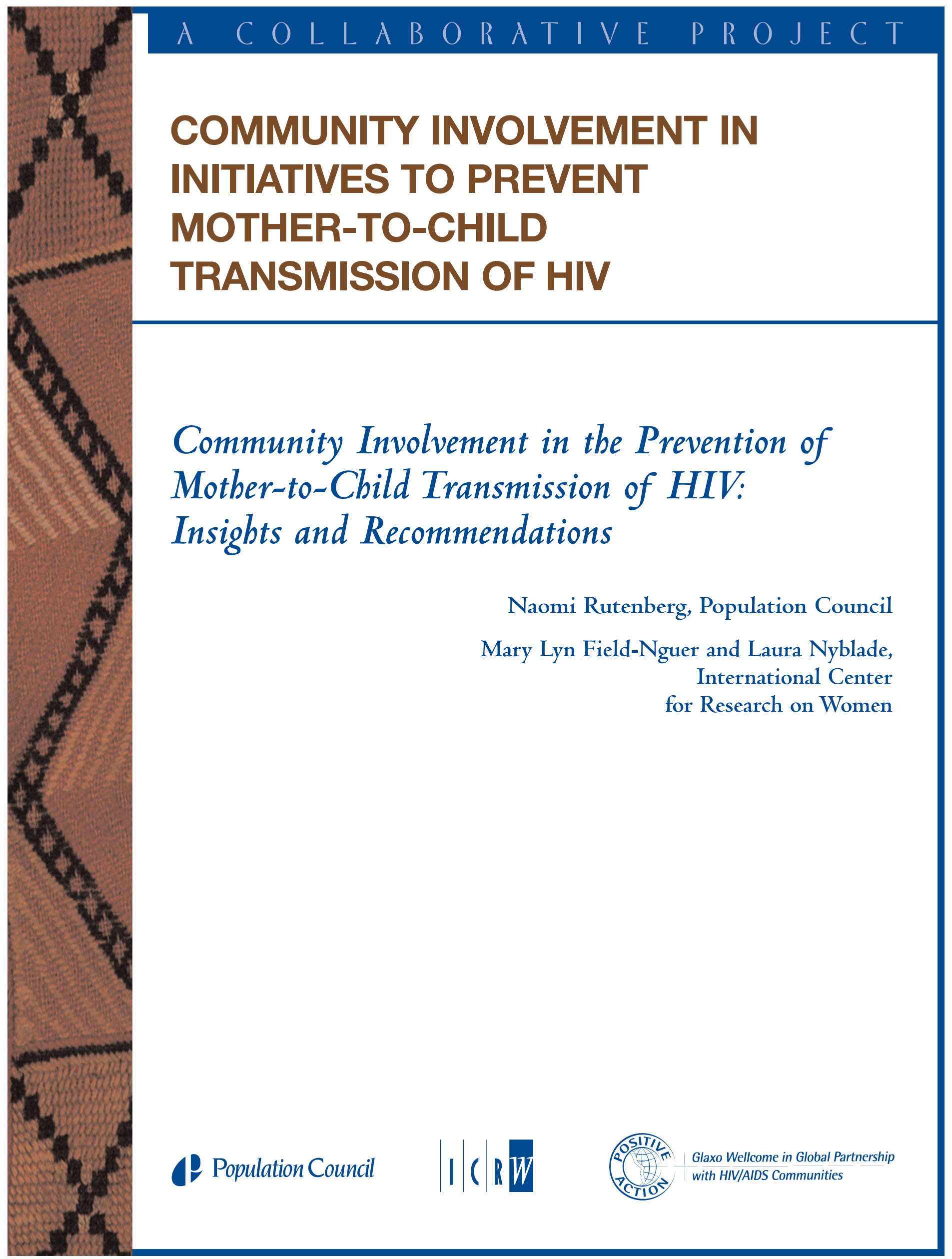




\title{
Community Involvement in the Prevention of Mother-to-Child Transmission of HIV: Insights and Recommendations
}

\author{
Naomi Rutenberg, Population Council; Mary Lyn Field-Nguer \\ and Laura Nyblade, International Center for Research on Women
}

Mother-to-child transmission is the primary route of HIV infection in children under 15 years of age. Since the beginning of the HIV epidemic, an estimated 5.1 million children worldwide have been infected with HIV (UNAIDS 2000). Clinical trials in several countries have shown that mother-to-child transmission of HIV can be greatly reduced through administering a short, affordable course of antiretroviral therapy to pregnant women. These trials culminated in a recommendation by UNAIDS and its partners in the Interagency Task Team for the Prevention of Mother-to-Child Transmission that prevention of perinatal transmission should be a part of the standard package of care for HIV-positive women and their children (UNAIDS 2000). Moreover, it is quickly becoming clear that prevention programs can enhance communities' understanding of and response to HIV. As a result, governments in Africa, Asia, and Latin America in collaboration with international and nongovernmental organizations have moved rapidly to improve antenatal care and incorporate interventions to prevent transmission of HIV from mother to child into clinical and community-based care.

The acceptability, operational concerns, costs, and impact of this package of services on preventing mother-to-child transmission of HIV and on child morbidity and mortality are being addressed through a number of monitoring and evaluation activities (see, for example, Leonard, Mane, and Rutenberg 2001). Equally important questions are being raised about how such interventions will be understood and received in the various communities in which they are becoming available, and how the interventions themselves can benefit from community input and involvement. In 1999 the Population Council and the International Center for Research on Women (ICRW) initiated several activities to identify effective mechanisms for enhancing community involvement in efforts to prevent mother-to-child transmission. With support from Glaxo Wellcome's Positive 
Action Program and UNAIDS, the Population Council and ICRW reviewed the literature on community involvement in the introduction of technologies and assessed community views on preventing mother-to-child transmission in Botswana and Zambia. In addition, the Horizons Program, implemented by the Population Council, ICRW, and others with support from USAID, has documented community responses to the introduction of such prevention services in Kenya and Zambia.

The research in Botswana and Zambia showed gaps in community knowledge about HIV transmission, particularly from mother to child, and yielded insights into community perspectives about the barriers to using voluntary counseling and testing services; the stigma and fear associated with HIV; traditional norms on breastfeeding; and the role of family and community members in women's decisions to participate in programs to prevent mother-to-child transmission of HIV (Nyblade and Field-Nguer 2001). Box 1 summarizes the key findings of this research. The literature review provided information about community involvement in earlier introductions of biomedical and other technologies. Such information can guide appropriate and effective community involvement (for a full discussion and examples, see Leonard, Mane, and Rutenberg 2001).

\section{Box 1. Selected findings from research in Botswana and Zambia}

- Women were better informed than men about all aspects of HIV/AIDS and transmission of HIV from mother to child. The researchers attribute this to the fact that maternal-child health clinics are the primary source of information about HIV/AIDS; men and community elders rarely make use of these clinic services.

- Misunderstandings about pregnancy and fetal blood supply were responsible for community members' doubts about the ability of drugs to prevent transmission of HIV from mother to child.

- The majority of study participants had little experience with counseling and testing services and thought that disclosure of testing, particularly if test results were positive, would be very difficult.

- People living with HIV/AIDS in the study communities face stigma, discrimination, and isolation, which are deterrents to taking advantage of HIV testing services.

- A key barrier to voluntary counseling and testing is the belief that the first member of a couple who is tested will be blamed for bringing HIV into the relationship.

- The strength of cultural norms and pressure to breastfeed was reflected in derogatory references to women who do not breastfeed.

- $\quad$ Partners, family, and community members strongly influence women's choices about voluntary counseling and testing and infant feeding.

Source: Nyblade and Field-Nguer 2001. 
By supporting community involvement, we can enhance prevention of mother-to-child transmission of HIV. Our recommendations are for the benefit of program managers, policymakers, and donors and apply to preventing vertical transmission of HIV in a number of settings, including prevention services for women who are likely to become pregnant and their partners. The recommendations acknowledge the right of people to know their serostatus and to access the information, services, and support they need to make decisions based on this knowledge. We hope that our recommendations will encourage program planners to include community participation, education, and mobilization as critical program elements.

\section{WHY COMMUNITY INVOLVEMENT?}

Greater community involvement in health services contributes to more effective, sustainable programs by providing a perspective that health services may otherwise lack. However, the benefits go beyond a better understanding of clients' needs, resources, and constraints. Forty years of development experience and interventions research show that programs that involve community members in developing, implementing, and monitoring activities are more likely to be acceptable to the community and to have more effective outcomes. Conversely, failure to involve the community may not only result in a failed intervention, but may also produce unforeseen and possibly adverse effects. In addition, community involvement can positively affect community norms and contextual factors to create an environment favorable to changes in behavior that may decrease the vulnerability of individuals and groups at risk within the community.

Engaging communities in preventing mother-to-child transmission is particularly challenging because of the complexity of the HIV transmission process and the stigma associated with HIV/AIDS. ICRW's research found that the benefits of technologies that prevent HIV infection (e.g., serostatus testing, antiretroviral drugs, and breastmilk substitutes) can be overshadowed by prevailing community norms, values, and beliefs regarding HIV/AIDS. For instance, the stigmatization of people living with HIV/AIDS compels those infected to hide their HIV-positive status and, when combined with 
misinformation about how HIV is transmitted and prevented, deters women from participating fully in efforts to prevent mother-to-child transmission. Overcoming negative community perceptions about people living with HIV/AIDS and educating community members about HIV transmission are necessary to increase women's willingness to be tested for HIV, a prerequisite for participating in interventions to prevent mother-to-child transmission.

The findings from Botswana and Zambia also clearly show that women trying to decide whether or not to participate in a program to prevent mother-to-child transmission of HIV are strongly influenced by the opinions of their spouses and partners, as well as those of family and community members. Parents, in-laws, and other relatives have varying degrees of influence on decisions about testing, disclosure, and drug treatment, and often have significant authority over infant feeding. The study findings demonstrate that, in order to succeed, programs to prevent mother-to-child transmission must be able to provide information, education, services, and support to these significant others in women's lives.

\section{WHEN TO INVOLVE THE COMMUNITY?}

A core element of programs to prevent mother-to-child transmission should be two-way communication between program staff and community members. Community knowledge and perspectives must be gathered and shared in order for program managers to design effective services and messages for the prevention of mother-to-child transmission of HIV. In addition, health services should maintain dialogue with the community to keep members informed about the purpose and availability of program services and to monitor acceptability and impact of the program within the community.

Programs that have not yet begun to implement interventions should begin with participatory research to ascertain how community norms affect attitudes and behavior regarding HIV/AIDS and mother-to-child transmission of HIV and to assess community perceptions of the need for information and services. Focus-group discussions and 
interviews with community members are an essential tool for both research and education. Those conducted as part of the studies in Botswana and Zambia highlighted the lack of community dialogue on many issues critical to effective programs for the prevention of HIV and mother-to-child transmission. This type of forum provides a unique opportunity for both community members and program managers to gain information and consider the complex challenges they face as a result of the AIDS epidemic. Most participants in the study were discussing these issues for the first time, a finding that underscores the fact that communities have long been passive recipients of, rather than active participants in, HIV/AIDS prevention efforts.

Programs in progress must establish these communication mechanisms as well, in order to facilitate free and open communication between community members and program staff, provide information, and provide a forum for discussing and monitoring the community's perceptions of the programs. Clear communication will also ensure that misconceptions, problems, and stigma associated with HIV/AIDS are addressed quickly.

\section{WHAT NEEDS TO BE DONE?}

While community participation need not require allocating substantial resources, it must include consistent and open-minded engagement throughout the intervention, with careful planning for how communities will continue to be engaged beyond the life of a specific project. Box 2 provides general guidelines for ensuring community involvement.

\section{Box 2. Guidelines for ensuring community involvement}

- Explore community norms and values.

- Assess and make use of existing community resources, including sources of social influence.

- Work through social networks.

- Foster family and social support.

- Harness the collective power of women as agents of change in the community.

- Create synergy between clinic- and community-based sources of information and services.

- Address the broader context within which community involvement is promoted.

Source: Leonard, Mane, and Rutenberg 2001. 


\section{Develop integrated communications strategies that include health education,}

evidence-based advocacy, social mobilization, counseling, and behavior change. Community dialogue and provision of information about HIV/AIDS and prevention of mother-to-child transmission are crucial to encouraging and sustaining participation in prevention programs. Program managers can make use of formative research and experience from other such programs to determine where HIV education is needed. For example, the research in Botswana and Zambia suggests that community education should include information on the probability of and factors associated with HIV transmission from mother to child during pregnancy and breastfeeding as well as how to live positively with HIV/AIDS. Pilot programs for preventing mother-to-child transmission revealed how service providers' attitudes toward HIV influence how prevention services are perceived and valued by their clients. Health providers have their own needs for information, education, and support that must not be neglected.

Preventing mother-to-child transmission of HIV requires behavior change at the individual and society level. Communication on preventing mother-to-child transmission should take place outside as well as inside the clinic walls. In addition, efforts should be directed not only at women, but also at those who influence women's decisions about counseling, testing, and infant feeding and those who influence policy decisions that guide the programs. Information from health care providers within the program should be reinforced by information from other sources and in a variety of venues.

\section{Introduce interventions to reduce stigma.}

Stigma associated with HIV/AIDS was a pervasive issue in the studies in Botswana and Zambia. Women reported fears related to disclosing their HIV-positive status, whether disclosure was voluntary or not (i.e., women might involuntarily disclose their status by using breastmilk substitutes or by initiating treatment). In addition, stigma has a significant influence on an individual's willingness to undergo HIV testing or to participate in other components of prevention programs. Program 
messages should address knowledge gaps and misconceptions about the prevalence of HIV infection, promote hope and compassion, and offer advice about and examples of positive living with HIV/AIDS.

Interventions must address stigma and discrimination against people living with HIV/AIDS, so that women can safely participate in programs to prevent mother-tochild transmission. In addition, support and services to families and community groups who care for people with AIDS are crucial so that those infected with HIV can be confident they will not be ostracized or abandoned and will receive the care they need.

\section{Foster social support for women.}

Programs should support the ability of women to make independent decisions. Principles of confidentiality and disclosure should be established in such a way that women are able to choose their own confidants and control the information they choose to share with others. Whether or not she involves a partner in decisions regarding prevention of mother-to-child transmission of HIV should be a woman's choice, and her right to make decisions without the involvement of her male partner must be respected. At the same time, we must maximize the opportunities for social support for pregnant, HIV-positive women. In some instances, women may feel more empowered when they receive social support from their peers.

\section{Go beyond HIV/AIDS.}

One of our goals is to mobilize communities to be open to innovation and behavior change. With that in mind, a viable entry point may be community concerns other than HIV/AIDS — for example, livelihoods, food security, or sanitation. Experience shows that once the process of change has begun, it spills across sectors. Examples from family planning, health, and agriculture show that women are not only amenable to new innovations but often emerge as leaders in the process of social change. Regardless of the particular activity, the key to involving women is to offer access to 
needed resources and opportunities in ways that are sensitive to the realities of their lives.

\section{Institutionalize a dynamic, participatory approach to monitoring and evaluation.}

Programs to prevent mother-to-child transmission of HIV are new, untested, and complex and will have to "learn by doing." Formative research provides an empirical basis for the initial design, but people's knowledge and attitudes change over timeoften rapidly after the introduction of services. There is a need, therefore, for a dynamic approach to monitoring and evaluating information provision, service design, and provider performance. It is critical that programs determine who is being reached by their messages and services, assess their acceptability in the community, and then act on this feedback. The feasibility of each of the program elements for clients, their families, providers, and the community should be continually monitored. An understanding of the acceptability of each program component-HIV counseling and testing, antiretroviral drugs, infant feeding counseling, and so forth-offers an opportunity to maximize participation, coverage, and impact.

\section{HOW TO DO IT?}

The research and experience of ICRW and the Population Council point to a number of approaches to effectively involving communities in programs to prevent mother-to-child transmission of HIV:

\section{Conduct formative research to obtain community-level data that can improve the acceptability and impact of services for preventing mother-to child transmission of HIV.}

Focus-group discussions provide a unique opportunity for participants to gain information and reflect on the complex issues they face as a result of the HIV epidemic. A strategy of participation, open discussion, and feedback should be the 
basis for research and education programs on HIV/AIDS and mother-to-child transmission.

2. Develop comprehensive, evidence-based communication strategies during the planning stages of pilot programs.

Strategies should address various layers of communication, including health education, evidence-based advocacy, social mobilization, counseling, and behavior change.

3. Encourage and foster peer education, peer counseling, and the establishment of support groups by building on existing community groups such as mothers' clubs, church groups, community health workers, and groups for people living with HIV/AIDS.

4. Meet community needs through programs other than those that address HIV/AIDS exclusively.

Expand interventions to include income-generating activities, support for kitchen gardens, child care cooperatives, and other solutions the community identifies as linked to the ability of women and men to change norms and behaviors related to HIV.

5. Establish community advisory committees that offer program managers and service providers feedback on how the program is being received and how it can be improved.

Not only can advisory committees provide guidance; they can be actively involved in all phases of program evaluation, including formative research to design services and messages, development of program indicators, analysis of the information collected, dissemination of information to program staff and the community, and advice and evidence-based advocacy to program managers. 


\section{WHO IS RESPONSIBLE?}

The success or failure of interventions that address mother-to-child transmission of HIV and their sustainability may well be determined by the extent to which resources are dedicated to community involvement. A community involvement strategy should be farreaching and long-term enough to have an impact. Government and international agencies that sponsor interventions should stimulate community involvement in three ways. First, program managers and donors must give community involvement the same high priority currently given to health facility-based interventions, such as the provision of supplies and provider training. Second, governments and donors must dedicate financial resources and mechanisms to support community involvement; communities should not be expected to shoulder the burden of participation alone. Finally, governments and donors must commit appropriate personnel to guide the implementation of formative research and integrated communications strategies and to assist efforts of community members to mobilize their resources, to lobby for change, and to participate.

\section{COMMUNITY INVOLVEMENT IS CRITICAL}

An intervention that addresses mother-to-child transmission of HIV is complex, yet it is one of the few biomedical interventions currently available for reducing the transmission of HIV that is feasible and affordable in resource-constrained settings. Placed within the framework of community involvement, it offers an enormous opportunity to improve HIV prevention and care. Successful interventions can influence how AIDS is perceived by the community, reduce stigma, and have an effect beyond the immediate prevention of perinatal transmission. Without community involvement, however, the opportunity to effect positive change may be squandered. 


\section{Acknowledgments}

The authors thank the following people for their ideas and guidance: George Brown, Ann Leonard, Purnima Mane, and Sue Perl, Population Council; Geeta Rao Gupta, International Center for Research on Women; and Shari Cohen and Eric Mercier, UNICEF.

\section{References}

Leonard, Ann, Purnima Mane, and Naomi Rutenberg. 2001. "Review of the evidence on the importance of community involvement: Implications for initiatives to prevent motherto-child transmission of HIV," in Community Involvement in Initiatives to Prevent Mother-To-Child Transmission of HIV: A Collaborative Project. New York: Population Council.

Nyblade, Laura and Mary Lyn Field-Nguer. 2001. "Women, communities, and the prevention of mother-to-child transmission of HIV: Issues and findings from community research in Botswana and Zambia," in Community Involvement in Initiatives to Prevent Mother-To-Child Transmission of HIV: A Collaborative Project. New York: Population Council.

UNAIDS. 2000. "Preventing mother-to-child HIV transmission: Technical experts recommend use of antiretroviral regimens beyond pilot projects," joint UNAIDS/WHO press release. Geneva: UNAIDS. 